\section{Operation im Bauchraum: Wie riskant ist sie für Schwangere?}

\begin{abstract}
Ein neuer Score soll helfen, das Risiko für Mutter und Kind bei notwendig werdenden chirurgischen Eingriffen im Bauchraum der Schwangeren abzuschätzen.
\end{abstract}

\begin{abstract}
$A$ ppendektomie und Cholezystektomie gehören zu den häufigsten nicht geburtshilflichen chirurgischen Eingriffen in der Schwangerschaft. Wie riskant solche Operationen für werdende Mutter und Kind sind, sollte eine retrospektive Kohortenstudie an 19.926 Schwangeren klären, denen in der Schwangerschaft entweder Blinddarm oder Gallenblase entfernt wurden. Nachfolgend kam es zu 1.018 unerwünschten Ereignissen hinsichtlich Schwangerschaft oder Geburt: Frühgeburt vor 37 . SSW (35,4\%), vorzeitige Wehen $(26,4 \%)$ und Fehlgeburt vor 22. SSW (25,7\%); zudem war die Klinikverweildauer länger (fünf vs. drei Tage) und das Sterberisiko höher (0,31 vs. $0,02 \%)$. Zehn Faktoren für einen ungünstigen
\end{abstract}

Schwangerschaftsverlauf wurden definiert. Im daraus entwickelten Score wurde für jeden Faktor risikoabhängig eine Punktzahl vergeben: Zervixinsuffizienz (16 Punkte), Vorwehen (15), Vaginitis oder Vulvovaginitis (8), Mehrlingsschwangerschaften bzw. Sepsis (6), offenes Operationsverfahren - im Gegensatz zur Laparoskopie - (5), Peritonitis (5), Drogenmissbrauch (3), ethnische Herkunft (2), Versichertenstatus - Medicare (1 Punkt).

Der Score wurde für einen „Übungsdurchlauf" mit drei Risikokategorien eingesetzt: niedriges Risiko für ein unerwünschtes Ereignis = max. 4 Punkte, intermediäres Risiko $=5-8$ Punkte, hohes Risiko $=9$ und mehr Punkte. Die Wahrscheinlichkeit einer Komplikation vor der
Klinikentlassung lag in den drei Kategorien bei $2,5 \%, 8,2 \%$ bzw. 21,8 \%. Danach wurde der Score an 7.971 Frauen validiert: $73,9 \%$ hatten ein niedriges, $21,1 \%$ ein mittleres und 5,1\% ein hohes Risiko. Die vorhergesagte Wahrscheinlichkeit für ein Ereignis lag in allen Gruppen innerhalb des $95 \%$-Konfidenzintervalls der tatsächlich beobachteten Wahrscheinlichkeit. Dies spricht den Autoren zufolge für die gute Qualität des Scores. Zumindest drei Risikofaktoren seien modifizierbar: operativer Zugangsweg, (Vulvo-)Vaginitis und Zervixinsuffizienz. So sei die Laparoskopie der offenen Operation bei Schwangeren möglichst vorzuziehen. Die Datenlage zur Therapie einer vaginalen Infektion im Vorfeld der Operation sei aber noch unzureichend, ebenso bei Maßnahmen wie Zervixzerklage oder Progesteron zur Therapie der Zervixinsuffizienz.

Dr. Elke Oberhofer

Sachs A et al. Risk Factors and Risk Stratification for Adverse Obstetrical Outcomes After Appendectomy or Cholecystectomy During Pregnancy. JAMA Surg 2017; online 18. Januar

\title{
Screening auf Brustkrebs: MRT steigert Genauigkeit
}

\section{Die Magnetresonanztomografie der Mammae verbessert die diagnostische Genauigkeit des Screenings auf Mammakarzinome erheblich. Vor allem Frauen mit dichtem Brustgewebe könnten profitieren.}

$\mathrm{m}$ Brustkrebsscreening ist die Magnetresonanztomografie (MRT) bisher meist ein Instrument, um Frauen mit hohem Risiko zu untersuchen. Ob sie auch bei Frauen mit durchschnittlichem Risiko (Lebenszeitrisiko 6-12\%) Vorteile gegenüber herkömmlichen Methoden hat, wurde in einer deutschen Studie untersucht.

Christiane Kuhl und Mitarbeiter bezogen 2.120 Frauen ein, die im herkömmlichen Screening (Mammografie \pm Ultraschall) den BI-RADS-Kategorien 1 oder 2 zugeordnet wurden (negativer oder gutartiger Befund). Zielgrößen waren die Raten zusätzlich entdeckter Tumoren und von Intervallkarzinomen, die Spezifität und der positive Vorhersagewert der Brust-MRT. Ausgewertet wurden 3.861 MRT. Bei 61 Frauen deck- te die MRT ein Mammakarzinom auf. 48 der Tumoren wurden im Prävalenz-, $13 \mathrm{im}$ Inzidenzscreening gefunden; einer davon im Mammografie-SonografieScreening. Es wurden also 60 Mammakarzinome allein mittels MRT gefunden: - alle, die bei Erstuntersuchung, und 12 von 13, die bei Folgeuntersuchungen aufgefallen waren. 20 waren duktale Insitu-Karzinome, 40 wuchsen invasiv. Sie waren im Median $8 \mathrm{~mm}$ im Durchmesser, 93,4\% Lymphknoten-negativ, 41,7\% (Prävalenzscreening) bzw. 46,0\% (Inzidenzscreening) hochgradige, entdifferenzierte Karzinome. Intervallkarzinome traten nicht auf.

Die Rate zusätzlich entdeckter Karzinome lag bei 15,5/1000 Screenings (Prävalenz: 22,6/1.000, Inzidenz: 6,9/1.000).
Bei den invasiven Tumoren beliefen sich die Detektionsraten auf 14,2/1.000 (Prävalenz) bzw. 6,3/1.000 (Inzidenz). Die Spezifität der MRT betrug $97,1 \%$, der positive Vorhersagewert 35,7\%; dieser lag damit zweieinhalbmal so hoch wie jener, der im jüngsten Evaluationsbericht zum Mammografiescreening mit $14 \%$ beziffert wurde. Die MRT kann also auch Frauen mit durchschnittlichem Brustkrebsrisiko nutzen - wären da nicht die Kosten. Laut Autoren könne die MRT die Zahl der abzuklärenden Befunde jedoch senken und dadurch Kosten sparen. Ein Problem bei der Mammografie ist die Brustgewebsdichte. Die Autoren verweisen darauf, dass mehr als die Hälfte der Frauen des Screenings Brustgewebe mittlerer und hoher Dichte hatte, was Sensitivität und Spezifität der Röntgenuntersuchung reduziert. Dies könne die hohe Rate an Intervallkarzinomen von 30-50 \% erklären, die mit dem MammografieScreening verknüpft ist. Dr. Robert Bublak

Kuhl C Et al. Supplemental Breast MR Imaging Screening of Women with Average Risk of Breast Cancer. Radiology 2017, online 21. Februar 